\title{
A novel mutation of interleukin-1 receptor antagonist (IL1RN) in a DIRA patient from Turkey: Diagnosis and treatment
}

\author{
Betül Sözeri ${ }^{1}$, Bengü Gerçeker-Türk², Başak Yıldız-Atıkan³ ${ }^{2}$ Sevgi Mir ${ }^{4}$, Afig Berdeli ${ }^{4,5}$ \\ ${ }^{1}$ Division Pediatric Rheumatology, Health Sciences University, Istanbul Umraniye Education and Research Hospital \\ Istanbul; Departments of ${ }^{2}$ Dermatology, ${ }^{3}$ Pediatric Infectious Diseases, ${ }^{4}$ Pediatric Rheumatology and ${ }^{5}$ Molecular Genetics, \\ Ege University Faculty of Medicine, Izmir Turkey. E-mail: betulsozeri@yahoo.com \\ Received: 30th July 2017, Revised: 8th October 2017, Accepted: 27th November 2017
}

SUMMARY: Sözeri B, Gerçeker-Türk B, Yıldız-Atıkan B, Mir S, Berdeli A. A novel mutation of interleukin-1 receptor antagonist (IL1RN) in a DIRA patient from Turkey: Diagnosis and treatment. Turk J Pediatr 2018; 60: 588-592.

Autoinflammatory diseases can cause severe inflammation in bone and skin such as neonatal-onset multisystem inflammatory disease (NOMID), Majeed syndrome, interleukin-36 receptor antagonist deficiency (DITRA) and deficiency of interleukin-1 (IL-1) receptor antagonist (DIRA) syndrome. Here we report a five-year old boy who was admitted to the hospital with pustular skin lesions and fever in the first month of his life. Molecular analysis of IL1RN gene revealed a single homozygous $C$ nucleotide deletion at nucleotide position 396 (p.Thr133Profs*118). The novel p.Thr133Profs*118 mutation found in our study caused frameshift mutation and as a result, the respective protein is most likely non-functional. The patient, who received a variety of treatments for various preliminary diagnoses until the final diagnosis (DIRA), was treated with recombinant IL-1Ra, anakinra, and experienced significant clinical improvement.

Key words: deficiency of interleukin-1 receptor antagonist, DIRA, mutation.

Autoinflammatory diseases (AID) are clinical disorders characterized by abnormally increased inflammation and mediated predominantly by effector cells and molecules of the innate immune system. ${ }^{1}$ Recurrent fever attacks are one of the hallmark symptoms of these diseases. Also what these diseases have in common is the presence of different skin manifestations and genetic transmission.

Interleukin-1 receptor antagonist (IL-1Ra) deficiency (DIRA) was described by GoldbachMansky and her colleagues ${ }^{2}$ in 2009 as an autosomal recessive autoinflammatory disease caused by mutations affecting the gene IL1RN encoding the endogenous IL-1 receptor antagonist. A mutation in the (IL1RN) gene produces a protein which lacks antagonistic activity of IL-1 receptor leading to a continuous activation of inflammatory pathways through IL-1, resulting in uninhibited production of the proinflammatory cytokines, IL-1a and $-1 \mathrm{~b} \cdot \mathbf{1}^{1,3,4}$

DIRA is a disease characterized with prominent dermatologic component like other IL-1- associated conditions such as the cryopyrinassociated periodic fever syndromes (CAPS), familial cold-induced autoinflammatory syndrome (FCAS), Muckle-Wells syndrome, and neonatal onset multisystem inflammatory disease (NOMID).5,6 All three disorders manifest in the skin as neutrophilic urticaria; but DIRA presents in the neonatal period with a severe pustular dermatitis (pustular psoriasis), skin pathergy, and nail dystrophy, also elevated acute-phase reactants, sterile osteomyelitis, and periostitis. ${ }^{2}$

Here in this report, we describe clinical and molecular data of a male child who had clinical signs of DIRA syndrome firstly analyzed in Turkey.

\section{Case Report}

A five-year old boy was admitted to the hospital with pustular skin lesions and fever in the first month of his life. He was born at 37 weeks to healthy no-consanguineous Turkish parents after an uncomplicated labor. During the first 
week of his life, he developed an erythematous and scaly eruption over the scalp and the perineum. The lesions appeared initially in his forehead and spread to the whole-body. He was referred to the Dermatology specialist for excessive skin lesions. Skin biopsy was performed and revealed signs of generalized pustular psoriasis (GPP). Skin biopsies showed intense neutrophilic epidermal and dermal infiltration and forming pustules with subcorneal localization. Acanthosis, spongiosis and exocytosis were shown in epidermis. Chemical bath with eau borique followed by liquid vaseline use was recommended. Also eye drops and local antibiotics were prescribed for conjunctivitis.

When the patient reached 15 month of age, he was transferred to our hospital due to loss of appetite and failure to thrive. He had continuous fever and no response to several antimicrobial therapies for sepsis, bacterial and fungal skin infections.

Besides his ill appearance, he had conjunctivitis, scurfy lesions on scalp, telangiectasia on cheeks and erythematous thoracic skin lesions in his examination. We failed to palpate the abdomen due to excessive abdominal distention. Left hand wrist was swollen, erythematous and tender with palpation and motion. Both hand and foot nails were dystrophic.

Laboratory investigations showed a hemoglobin $(\mathrm{Hb})$ level of $7.3 \mathrm{~g} / \mathrm{dl}$, a white blood cell (WBC) count of 12,980 cells $/ \mathrm{mm}^{3}$ with $88 \%$ neutrophils, a platelet count of 980,000 cells $/ \mathrm{mm}^{3}$, an erythrocyte sedimentation rate (ESR) of $81 \mathrm{~mm} /$ hour in the first hour, and a C-reactive protein (CRP) level of $11.3 \mathrm{mg} /$ dl. Immunoglobulin levels were measured respectively as $\operatorname{IgG} 1,456 \mathrm{mg} / \mathrm{dl}$, IgA $116 \mathrm{mg} /$ $\mathrm{dl}$ and IgM $153 \mathrm{mg} / \mathrm{dl}$.

All blood and urine cultures were negative, so we decided to stop the antibiotherapy started for previous sepsis attack. During follow-up, Omenn sydrome and Netherton syndrome were taken into consideration but lymphocyte panel was normal and even serum IgE level was high. His hair strand analysis was negative excluding the Netherton syndrome.
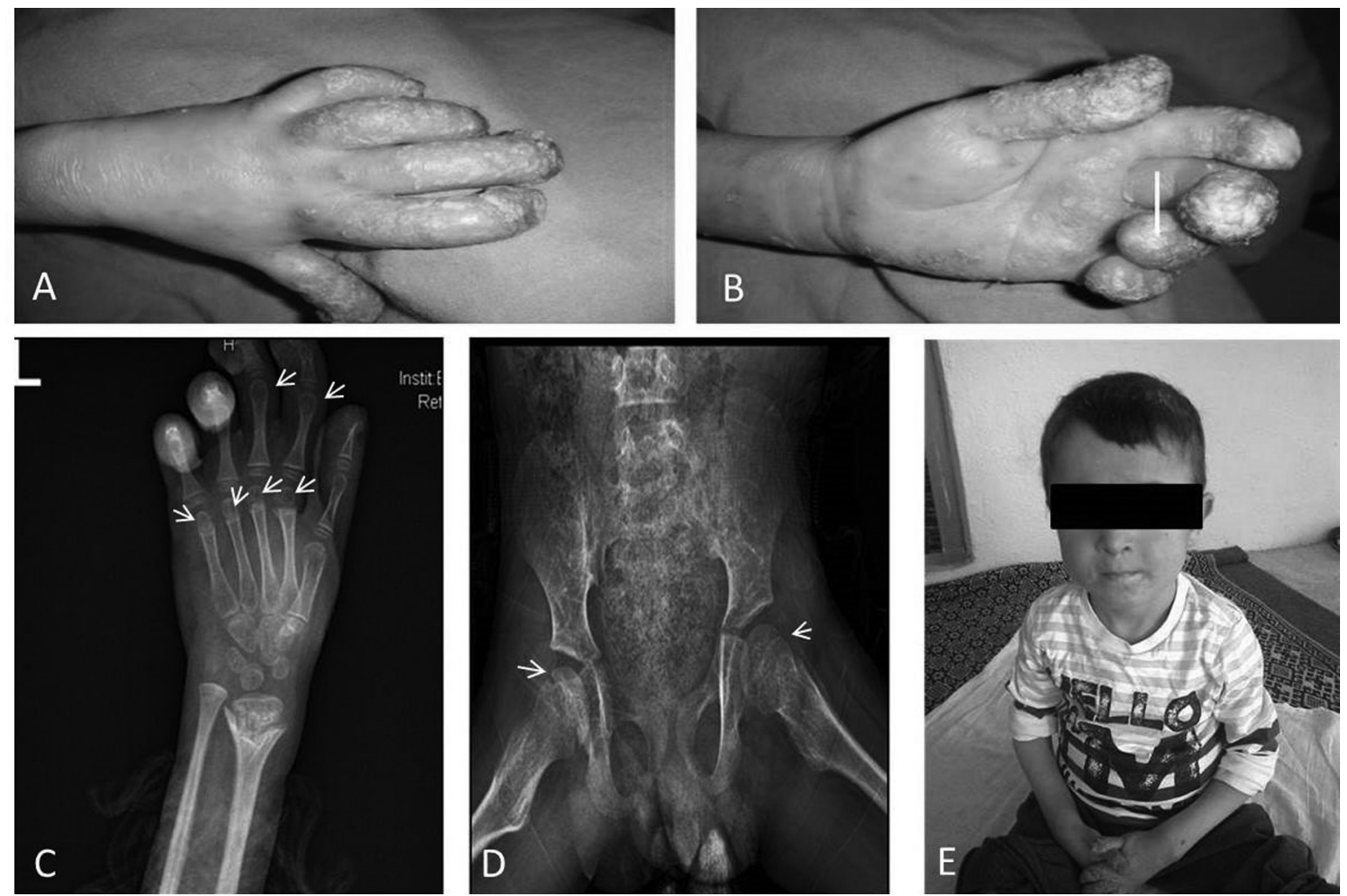

Fig. 1. Patient before anakinra treatment: The skin manifestations range from groupings of small pustules (A,E) to a generalized pustulosis (B). The bone manifestations include epiphyseal ballooning of multiple distal and proximal long bones (C and D); periosteal elevation of the diaphysis (arrowheads) (D). 

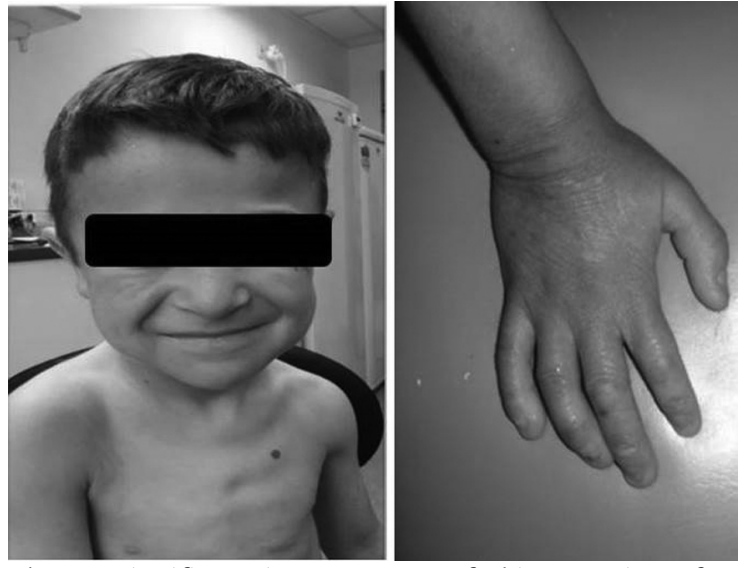

Fig. 2. Significant improvement of skin eruption after 1 month of anakinra treatment of erythema, complete vanishing of scales and pustules.

Severity of inflammation on skin lesions were altered during follow-up and pathological examination of the skin biopsy during exacerbation revealed similar findings with pustular psoriasis. Topical and systemic steroids and also broad spectrum antibiotics were used to treat sepsis and inflammation. $\mathrm{He}$ was given intravenous immunoglobulin replacement therapy several times. But there was no response to any of these therapies and methotrexate was given (1 to $3.5 \mathrm{mg} /$ week). Systemic steroids and cyclosporine (2 $\mathrm{mg} / \mathrm{kg}$ /day) were used when methotrexate was not efficient to prevent exacerbations and recurrent infections due to damage on the skin. In the 33rd month of his follow up, he had developed high grade fever, tachycardia (160$170 \mathrm{bpm})$ and massive hepatomegaly $(7 \mathrm{~cm}$ below costal margine) despite treatment with broad spectrum antibiotics. Echocardiography was performed to exclude cardiac insufficiency but there were no findings except for left ventricular hypertrophy. He complained of pain and swelling on right knee, left wrist and all metacarpophalangeal joints. Osteitis in his left distal radius, acetabulum and several ribs was identified by X-ray. In addition to that, systemic inflammation could not be stopped.

His laboratory tests indicated mild anemia, high WBC and platelet counts, and elevated CRP levels $(23.7 \mathrm{mg} / \mathrm{dl})$.

Asitretin therapy $(10 \mathrm{mg} / \mathrm{d})$ was started but was proved to be ineffective and further differential diagnosis and treatment modalities were needed. Etanercept therapy was started due to psoriatic arthritis and benefits were limited.

As this patient had presented with repeated skin flares early after birth, as well as serious constitutional distress with failure to thrive and arthritis, an autoinflammatory syndrome like interleukine-1-receptor antagonist deficiency (DIRA) was considered.

Molecular analysis of IL1RN gene was done at Ege University, Department of Moleculer Medicine, Izmir, Turkey. Genomic DNA was obtained from peripheral blood. All exon and intron of IL1RN gene were analyzed by PCR and direct DNA sequencing method. Furthermore, the obtained nucleotide sequence was compared with reference sequence published in NCBI (NM_173841.2). We found a single homozygous C nucleotide deletion at nucleotide position 396 (c.396delC) in ILRN gene by DNA sequencing analysis. The novel c.396delC mutation that was found in our study caused frameshift mutation and as a result, stop codon of IL1RN at c.534* position disappeared and the respective protein became non-functional. Our result is currently accepted as a novel mutation in DIRA syndrome by database INFEVERS. ${ }^{7}$ Also, we found a polymorphism (c.399T $>$ C, homozygous) in the gene.

Recombinant IL-1-receptor antagonist, anakinra at $2 \mathrm{mg} / \mathrm{kg} /$ day was started with rapid effectiveness on all symptoms (disappearance of irritability, skin involvement and arthritis) (Fig. 1 and 2). Before treatment he could not walk and had to use a wheelchair and he began walking after treatment.

\section{Discussion}

We presented a patient diagnosed with deficiency of the interleukin-1- receptor antagonist, who had osteitis, arthritis and pustulosis at the first week of his life. Children with DIRA present with strikingly similar clinical features, including systemic inflammation in the perinatal period, bone pain, characteristic radiographic findings of multifocal sterile osteolytic bone lesions, widening of multiple anterior ribs, periostitis, pustular skin lesions, skin pathergy, and elevated acute phase reactants. ${ }^{2,4}$ Affected individuals show symptoms like fetal distress, pustular rash, joint swelling, oral lesions and pain with movement between birth and 2.5 weeks of age. ${ }^{6}$ Fever is typically absent. 
In our patient, an erythematous and scaly eruption was since $1^{\text {st }}$ week of life, while osteolytic bone lesions periostitis and nail changes were determined in $15^{\text {th }}$ month of age.

The patient was initially diagnosed with pustular psoriasis based on clinical presentation and biopsy results. Various inflammation and generalized pustulosis to ichthyosiform lesions in the skin has been present in $95 \%$ of the affected patients. ${ }^{3}$ This severe pustular skin rash, that usually effects palms and soles, may result in diffuse desquamation. Nail deformations are common in DIRA. Severe rash and nail changes in our patient findings were shown in Figure 1. Bony changes include epiphyseal ballooning of long bones, anterior rib end widening, periosteal elevation of long bones, and multifocal osteolytic lesions were reported in the DIRA patients, previously. 2,4,10,11 In the literature, half of infants with DIRA have been born prematurely (31 to 36 weeks' gestation)..$^{2,4,8,9}$ Our patient was born at 37 weeks to healthy non-consanguineous Turkish parents. Respiratory insufficiency and thrombotic events were seen in patients reported previously ${ }^{2,4,8-12}$, was not apparent in our patient.

To date, fewer than 20 cases from the United States, Canada, the Netherlands, Brazil and Puerto Rico have been described. First generation mutations in these distinct geographic populations are believed to be founder mutations. The allele frequencies of the founder mutations in Newfoundland and Puerto Rico are estimated at $0.2 \%$ and $1.3 \%$, respectively.

In INFEVERS database, 15 founder ILRN gene mutation has been reported from different countries ${ }^{7}$; Puerto Rico (c. 64 1696del in5'UTR), Morocco (c.116+34G>A) $(c .390 T>C)$ (c. $\left.{ }^{*} 138 C>G\right)$, Canada (c.156 157del), Lebanon $(c .160 C>T)\left(c .{ }^{*} 162 C>T\right)$, Brazil (c.213 227del), Holland $(c 229 G>T)$, Germany $(c .2 \overline{7} 2 G>T)$, Turkey $(c 355 C>T)$ and unknown origin $(c 171 T>C)$. Among these patients 6 had DIRA phenotype while 7 patients had other auto inflammatory diseases. ${ }^{7}$ We found a single homozygous $\mathrm{C}$ nucleotide deletion at nucleotide position 396 (c.396delC) in ILRN gene. The novel c.396delC mutation that was found in our study caused frameshift mutation and as a result, stop codon of IL1RN at c.534* position disappeared and the respective protein became non-functional. Our result was accepted as novel mutation in DIRA syndrome by database INFEVERS.

Recombinant IL-1-receptor antagonist, anakinra at $2 \mathrm{mg} / \mathrm{kg} /$ day was started with rapid effectiveness on all symptoms (disappearance of irritability, skin involvement and arthritis) (Fig. 2). Before treatment he could not walk and had to use a wheelchair and began walking after treatment. In the literature, Jesus A et al. ${ }^{10}$ reported that partial response with steroid in two Brazilian patients with DIRA. Another infant with generalized pustular psoriasis recovered her skin lesion with acitretin. ${ }^{13}$ Besides Aksentiewitz et al. ${ }^{2}$ reported that they received a good response with anakinra, a recombinant IL-1Ra, in treatment of 6 patients.

The patient, who used a variety of treatments for various preliminary diagnoses until the correct diagnosis (DIRA), was treated with recombinant IL-1Ra, anakinra, and experienced significant clinical improvement. It is the first case that the accurate genetic diagnosis of a case considered as DIRA has been confirmed with whole sequence analysis of IL1RN gene in Turkey.

\section{REFERENCES}

1. Kastner DL, Aksentijevich I, Goldbach-Mansky R. Autoinflammatory disease reloaded: A clinical perspective. Cell 2010; 140: 784-790.

2. Aksentijevich I, Masters SL, Ferguson PJ, et al. An autoinflammatory disease with deficiency of the interleukin-1-receptor antagonist. N Engl J Med 2009; 360: 2426-2437.

3. Naik HB, Cowen EW. Autoinflammatory pustular neutrophilic diseases. Dermatol Clin 2013; 31: 405425.

4. Reddy S, Jia S, Geoffrey R, et al. An autoinflammatory disease due to homozygous deletion of the IL1RN locus. N Engl J Med 2009; 360: 2438-2444.

5. Goldbach-Mansky R, Kastner DL. Autoinflammation: The prominent role of IL-1 in monogenic autoinflammatory diseases and implications for common illnesses. J Allergy Clin Immunol 2009; 124: 1141-1149.

6. Cowen EW, Goldbach-Mansky R. DIRA, DITRA, and new insights into pathways of skin inflammation: What's in a name? Arch Dermatol 2012; 148: 381-384.

7. Infevers: an online database for autoinflammatory mutations. Available at: http://fmf.igh.cnrs.fr/ISSAID/ infevers/ (Accessed: 22nd February 2019)

8. Altiok E, Aksoy F, Perk Y, et al. A novel mutation in the interleukin-1 receptor antagonist associated with intrauterine disease onset. Clin Immunol 2012; 145: 77-81. 
9. Minkis K, Aksentijevich I, Goldbach-Mansky R, et al. Interleukin 1 receptor antagonist deficiency presenting as infantile pustulosis mimicking infantile pustular psoriasis. Arch Dermatol 2012; 148: 747-752.

10. Jesus AA, Osman M, Silva CA, et al. A novel mutation of IL1RN in the deficiency of interleukin-1 receptor antagonist syndrome: Description of two unrelated cases from Brazil. Arthritis Rheum 2011; 63: 40074017.

11. Stenerson M, Dufendach K, Aksentijevich I, Brady J, Austin J, Reed AM. The first reported case of compound heterozygous IL1RN mutations causing deficiency of the interleukin-1 receptor antagonist. Arthritis Rheum 2011; 63: 4018-4022.
12. Schnellbacher C, Ciocca G, Menendez R, et al Deficiency of interleukin-1 receptor antagonist responsive to anakinra. Pediatr Dermatol 2013; 30: 758-760.

13. Ergin S, Ersoy-Evans S, Sahin S, Ozkaya O. Acitretin is a safe treatment option for infantile pustular psoriasis. J Dermatolog Treat 2008; 19: 341-343. 